\title{
CARACTERIZAÇÃO ESPACIAL DE UM ÍNDICE DE PRODUTIVIDADE NOS POVOAMENTOS DE PINHEIRO-BRAVO EM PORTUGAL
}

\author{
CRISTINA SANTOS ${ }^{1}$ \\ José AlmeIdA 2
}

\begin{abstract}
Resumo - O planeamento sustentado dos recursos florestais é, hoje, considerado um imperativo fundamental na actual conjuntura económica portuguesa. Neste sentido existe uma necessidade premente de metodologias de modelação inovadoras e suficientemente robustas, para que as decisões estratégicas sejam tomadas com fundamentação técnica e científica.

O principal objectivo deste trabalho consiste na inferência espacial de um índice de produtividade do pinheiro-bravo (Pinus pinaster Ait.) na floresta portuguesa (este índice é apresentado em classes), utilizando metodologias geoestatísticas de simulação e funções de análise espacial. Estas metodologias permitem estimar este índice, para os locais onde ocorre esta espécie, mas onde não se procedeu à recolha de informação de campo, e associar um grau de incerteza.
\end{abstract}

Palavras-chave : Índice de produtividade, pinheiro-bravo, simulação geoestatística, funções de análise espacial, incerteza local, Portugal.

\begin{abstract}
SPATIAL CHARACTERIZATION OF A PRODUCTIVITY INDEX OF MARITIME PINE IN PoRTugal. Forestry plays an important role in the Portuguese regional economy. With this in mind, it is clear that robust and consistent modelling techniques are required so that all decisions can be scientifically based.

This work aims to calculate maps illustrating the spatial distribution of the productivity index of maritime pine in Portuguese forests, using geostatistical algorithms of stochastic simulation in association with GIS and spatial analysis functions. These geostatistical algorithms enable this productivity index to be inferred for the locations where this species occurs, but without sampling observations.

In this particular case study, data were provided by the third National Forest Inventory of the State Forest Administration. Based on measurements collected in
\end{abstract}

1 Engenheira Florestal. Direcção de Serviços de Planeamento e Estatística, Direcção-Geral das Florestas, Avenida João Crisóstomo, n. ${ }^{\circ}$ 26-28, 1069-040 Lisboa. Tel: +351 213124862 . Fax: +351213 124 983. E-mail: c.santos@darf.min-agricultura.pt

2 CIGA/FCT, Universidade Nova de Lisboa. E-mail: ja@fct.unl.pt 
sampling plots, of the height and age of dominant trees, a productivity index was calculated, summarizing the growth gradient level per year.

Experimental variograms showed that the productivity index treated as a categorical variable with five classes does not reveal any spatial continuity. On the other hand, the two components of the index - height and age of the trees - present a spatial structure displayed by long-range variograms. Hence, instead of dealing with the productivity index, the decision was made to characterize the spatial distribution of these two factors and to compose the index as the final step. Thus, a methodology is used for the joint spatial characterization of the height and age of maritime pine prior to the calculation of a productivity index.

One of the main advantages of stochastic simulation methodologies is the ability to assess the local uncertainty (related to sampling density, location of the nearest samples and local variability), as well as to map the probability of occurrence of extreme scenarios (in this case study, for instance, excellent or unproductive areas). These methodologies are major tools for the sustainable planning and management of natural resources, making it possible to delimit areas of greater and lesser suitability for each type of forest and to understand better the influence of factors that really affect tree development and growth.

Key words : Quality index, maritime pine, geostatistical simulation, spatial analysis functions, local uncertainty, Portugal.

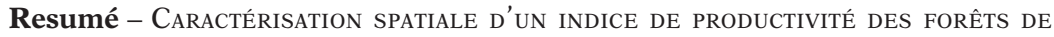
PINS MARITIMES AU PORTUGAL. La planification durable des ressources forestières est une nécessité dans la conjoncture économique actuelle du Portugal. Elle implique l'adoption de nouvelles techniques de modélisation, qui permettent de donner des bases techniques et scientifiques correctes à la prise de décision.

L'objectif de ce travail est l'élaboration de cartes de distribution spatiale des classes de productivité du pin maritime, par l'utilisation d'une méthode géostatistique de simulation, permettant de déterminer les classes de productivité et leur degré d'approximation, aux endroits où des observations de terrain n'ont pas été réalisées.

On utilise pour cela le troisième Inventaire Forestier National (1999). En partant de donnés sur la hauteur et l'âge des pins, on détermine cinq classes de productivité annuelle et leur degré de variabilité. On peut ainsi situer les secteurs les mieux adaptés aux divers types de forêt et comprendre les facteurs qui conditionnent la croissance des arbres.

Mots-clés : Classes de productivité, pin maritime, simulation géostatistique, incertitude locale, Portugal.

\section{INTRODUÇÃO}

Actualmente, a floresta - e em particular a de pinheiro-bravo (Pinus pinaster Ait.) -, para além do valor dendrológico, histórico e social, tem uma contribuição fundamental para a economia do país e para a qualidade ambiental. Em Portugal, o pinheiro-bravo é a resinosa autóctone mais representativa, ocupando uma área de cerca de $31 \%$ do coberto florestal (cerca de 1 milhão de ha) localizando-se essencialmente no Norte e Centro do país. No entanto, a 
sua distribuição geográfica é determinada por factores que impõem limitações severas ao seu crescimento (Oliveira et al., 2000), nomeadamente as condições edáficas, bioclimáticas e antrópicas.

Os recursos florestais são avaliados periodicamente com base em inventários florestais, que constituem, simultaneamente, um dos meios de monitorização da sustentabilidade da floresta no tempo. No âmbito da $3{ }^{a}$ Revisão do Inventário Florestal Nacional (Direcção-Geral das Florestas, 2001), efectuou-se, numa primeira fase e para todo o país, a avaliação qualitativa do tipo de ocupação do solo, a partir de um mosaico de fotografias aéreas ${ }^{3}$. $\mathrm{O}$ suporte desta avaliação preliminar é constituído por uma rede regular de pontos de amostragem, designados por fotopontos. Na segunda fase, e tendo como objectivo a avaliação dos parâmetros ao nível dos povoamentos florestais, efectuou-se uma recolha de dados no campo por observação de pequenas áreas em parcelas de amostragem, distribuídas aleatoriamente por todo o território. Ao todo foram foto-interpretados 134550 pontos, dos quais 49446 foram classificados com o atributo ${ }^{4}$ florestal e onde foram classificados 12877 pontos como floresta de pinheiro-bravo. Deste conjunto foram seleccionados aleatoriamente 795 pontos, procedendo-se à sua marcação nas fotografias aéreas, para verificação do estrato e posterior medição de parâmetros dendrométricos em levantamentos de campo. As medições incluem a idade e o diâmetro à altura do peito (diâmetro do tronco da árvore medido à altura de $1,30 \mathrm{~m}$ do solo), diâmetro basal e altura total. Estes parâmetros permitem inferir cálculos que ajudam a avaliar povoamentos florestais, nomeadamente através da construção de índices que sumariam a produtividade e permitem a comparação entre povoamentos.

O principal objectivo deste trabalho consiste na elaboração de um mapa do índice de produtividade $(I P)$ do pinheiro-bravo em Portugal e num correspondente mapa de incerteza. A localização das regiões de melhor produtividade é um dado fundamental, face à tão desejada optimização da rentabilidade das matérias-primas, tendo sempre por base a sustentabilidade da fileira do pinho, na sua vertente económica, social e ambiental.

\section{METODOLOGIA ADOPTADA}

\section{1. Índice de produtividade}

Vários autores propõem a avaliação da produtividade baseada na relação da altura das árvores para uma determinada idade de referência. Neste estudo foi utilizado um índice, que relaciona a altura das árvores dominantes $\left(H_{d o m}\right.$

3 Optou-se por utilizar um filme fotográfico do tipo infravermelho de falsa-cor, que permite uma distinção mais evidente entre espécies folhosas e espécies resinosas.

4 De acordo com a metodologia da $3 .^{\text {a }}$ revisão do IFN, entende-se por atributo ou «mancha» a porção de terreno, de área igual ou superior a 0,5ha e de largura média igual ou superior a $20 \mathrm{~m}$, que constitui uma unidade homogénea do ponto de vista do uso e ocupação do solo. 
- variável altura dominante) com a idade correspondente ( $I$ - variável idade). $\mathrm{O}$ índice é construído com base na inferência da altura para a idade de referência de 50 anos $\left(H_{50}\right)$ utilizando o método das Curvas Guia (Alves, 1988). Este índice $(I P)$ é codificado com valores entre 1 e 5 , consoante o intervalo da altura (Quadro I),

$$
\begin{gathered}
H_{50}=\frac{20 H_{\text {dom }}}{30,600068 \times 10^{(-9,2350557 / I)}} \\
I P=7-I N T\left(H_{50} / 4+0,05\right)
\end{gathered}
$$

em que $I N T(x)$ é uma função que arredonda $x$ para o inteiro mais próximo.

Quadro I - Índice de produtividade (IP) da estação dos povoamentos de pinheiro-bravo.

Table I-Productivity index (IP) of maritime pine areas.

\begin{tabular}{cll}
\hline $\boldsymbol{I P}$ & $\boldsymbol{H}_{\mathbf{5 0}}-$ altura projectada aos $\mathbf{5 0}$ anos de idade & Designação \\
\hline 1 & $H_{50}>240 \mathrm{dm}$ & Alta \\
2 & $200 \mathrm{dm}<H_{50} \leq 240 \mathrm{dm}$ & Média-alta \\
3 & $160 \mathrm{dm}<H_{50} \leq 200 \mathrm{dm}$ & Média \\
4 & $120 \mathrm{dm}<H_{50} \leq 160 \mathrm{dm}$ & Média-baixa \\
5 & $H_{50} \leq 120 \mathrm{dm}$ & Baixa \\
\hline
\end{tabular}

\section{Construção do mapa do índice de produtividade}

Para a construção do mapa das classes do índice de produtividade $(I P)$, para o pinheiro-bravo a nível nacional, a metodologia adoptada resume-se às seguintes etapas:

a) Análise exploratória de dados das variáveis altura dominante e idade;

b) Cálculo dos variogramas experimentais para as variáveis altura dominante e idade e ajustamento de modelos teóricos;

c) Simulação estocástica de dez imagens da variável altura dominante, utilizando o método da Simulação Sequencial Directa;

d) Simulação estocástica de dez imagens da variável idade, utilizando o método da Co-Simulação Sequencial Directa;

e) Construção de dez mapas simulados das classes do índice de produtividade do pinheiro-bravo, apenas para as áreas do país onde é confirmada a presença desta espécie pela foto-interpretação;

f) Construção do mapa final das classes do índice de produtividade mais provável em cada ponto;

g) Determinação de um mapa de incerteza, que fica associado ao mapa do índice. 
Para a elaboração da carta nacional do índice de produtividade do pinheiro-bravo, optou-se por efectuar a modelação das variáveis altura das árvores dominantes $\left(H_{d o m}\right)$ e a idade correspondente $(I)$ separadamente, procedendo-se à construção do índice a posteriori. A única razão deste procedimento deve-se ao facto de o índice calculado com base no método das Curvas Guia para os pontos experimentais não apresentar continuidade espacial significativa. Por outro lado, as variáveis (altura dominante e idade) necessárias à caracterização do índice de produtividade apresentam estrutura espacial bem definida, revelada nos variogramas experimentais. Assim, em vez de se trabalhar directamente com a variável índice de produtividade, decidiu-se caracterizar separadamente a distribuição espacial destas duas variáveis por simulação e compor o índice num mapa final, aplicando as equações (1) e (2) célula a célula.

\section{Simulação estocástica}

Os modelos de simulação estocástica têm como objectivo a elaboração de imagens 'virtuais' da realidade, que reflectem as características do recurso natural, a variabilidade do conjunto de amostras, a lei de distribuição da variável em estudo e a continuidade espacial.

Com estes métodos de simulação, não se pretende obter a imagem mais provável das características de um dado recurso, mas sim um conjunto de imagens equiprováveis ${ }^{5}$ com a mesma variabilidade espacial dos valores experimentais. Este conjunto de imagens permite visualizar o comportamento extremo das características internas ou morfológicas de um dado recurso natural e, simultaneamente, quantificar a incerteza da localização espacial dessas características. A incerteza espacial é calculada através de medidas estatísticas da variabilidade do conjunto de imagens equiprováveis do fenómeno espacial.

Existem vários algoritmos de simulação candidatos a gerar imagens das variáveis altura dominante e idade, onde se destacam os algoritmos de simulação sequencial. Neste trabalho foi utilizado o algoritmo de Simulação e Co-simulação Sequencial Directa (SSD). Este algoritmo de simulação sequencial de imagens difere da Simulação Sequencial Gaussiana (SSG) clássica por não requerer uma transformação prévia das variáveis originais para uma distribuição do tipo gaussiano (GoovAERTs, 1997), assumindo, por isso, vantagens notórias na simulação conjunta de variáveis.

A simulação estocástica de imagens da variável altura dominante $-Z_{1}(x)$ foi efectuada através do método de Simulação Sequencial Directa. A simulação da idade $-Z_{2}(x)$ foi efectuada posteriormente através do método de Co-Simulação Sequencial Directa, utilizando-se cada imagem simulada da altura dominante como imagem secundária (ou soft). Este procedimento deve-se à existência de

5 Imagens com os mesmos estatísticos de variabilidade inferidos pelos dados experimentais. Em cada célula, os valores possuem a mesma probabilidade de ocorrência. 
elevada correlação entre estas duas variáveis (quantificada em 0,80 pelo Coeficiente de Correlação de Pearson). A ordem de simulação (primeiro a altura dominante e depois a idade) é justificada pela maior incerteza nas medições experimentais da idade, corroborada pela menor continuidade estrutural do variograma. Posteriormente, todos os pares de imagens de valores simulados de $Z_{1}^{S}(x)$ e $Z_{2}^{S}(x)$ são compostos numa combinação de mapas simulados do índice para as áreas de pinheiro-bravo, tendo por base as equações (1) e (2) célula a célula.

A partir dos vários mapas simulados e equiprováveis do índice, pode representar-se em cada ponto o valor do índice mais provável, assim como calcular uma medida de variabilidade, também, célula a célula.

\section{Síntese teórica dos algoritmos de Simulação e Co-Simulação Sequencial Directa}

Se escolhermos $Z_{1}(x)$ como variável primária, ou seja, a variável mais importante ou que evidencia uma maior continuidade espacial, a Simulação e Co-simulação Sequencial Directa é resumida nas seguintes etapas (SOARES, 2001):

a) Escolha de uma sequência aleatória de células de uma malha regular, que permita a visita única de todas as células.

b) Em cada célula $x_{u}$ simulação do valor de $z^{s}{ }_{1}\left(x_{u}\right)$, utilizando o algoritmo de Simulação Sequencial Directa:

- Estimação da média e variância de $z_{1}(x), z_{1}\left(x_{u}\right) *$ e $\sigma_{s k}^{2}\left(x_{u}\right)$ por krigagem simples;

- Re-amostrar localmente o histograma de $z_{1}\left(x_{u}\right)$, utilizando, por exemplo, uma transformação gaussiana $\left(\varphi_{1}\right)$ da variável primária $Z_{1}(x)$; cálculo de $y\left(x_{u}\right)^{*}=\varphi_{1}\left[z\left(x_{u}\right)^{*}\right]$;

- Geração de um número aleatório $p$, a partir de uma lei uniforme U entre $[0,1]$;

- Geração do valor $y^{s}$ a partir de $G\left(y\left(x_{u}\right)^{*}, \sigma_{s k}^{2}\left(x_{u}\right)\right)$ :

$$
y^{s}=G^{-1}\left(y\left(x_{u}\right)^{*}, \sigma_{s k}^{2}\left(x_{u}\right), p\right) ;
$$

- Retorno do valor simulado da variável primária $z_{1}^{s}\left(x_{u}\right)=\varphi^{-1}{ }_{1}\left(y^{s}\right)$.

O mesmo algoritmo é aplicado, seguidamente, para a simulação da variável secundária $z_{2}^{s}\left(x_{u}\right)$, utilizando, agora, o estimador krigagem simples co-localizada $^{6}$ (requer o variograma da variável secundária e o coeficiente de corre-

6 Este estimador de krigagem simples co-localizada utiliza os valores da variável secundária na vizinhanca de $x_{u}$ e o valor da variável primária, previamente simulado em $x_{u}$. 
lação entre as variáveis primária e a secundária) para a estimação de $z_{2}\left(x_{u}\right)$ * e $\sigma_{s k}^{2}\left(x_{u}\right)$ :

$$
z_{2}\left(x_{u}\right){ }_{s c k}=\sum_{\alpha=1}^{N} \lambda_{\alpha}\left(x_{u}\right)\left[z_{2}\left(x_{\alpha}\right)-m_{2}\right]+\lambda_{\beta}\left(x_{u}\right)\left[z_{1}\left(x_{\alpha}\right)-m_{1}\right]+m_{2}
$$

A partir daqui, o algoritmo segue um procedimento idêntico ao que foi definido para a variável primária, ou seja:

- Cálculo de $y\left(x_{u}\right)^{*}=\varphi_{2}\left(z_{2}\left(x_{u}\right)^{*}\right)$, em que $\varphi_{2}$ é a transformada gaussiana da variável secundária $Z_{2}(x)$;

- Geração de um valor $p$ a partir de uma lei uniforme U entre $[0,1]$;

- Geração do valor $y^{s}$ a partir de $G\left(y_{2}\left(x_{u}\right)^{*}, \sigma^{2}{ }_{s c k}\left(x_{u}\right)\right)$ :

$$
y^{s}=G^{-1}\left(y_{2}\left(x_{u}\right)^{*}, \sigma_{s c k}^{2}\left(x_{u}\right), p\right) \text {; }
$$

- Retorno do valor simulado da variável secundária $z_{2}^{s}\left(x_{u}\right)=\varphi^{-1}{ }_{2}\left(y^{s}\right)$;

- Continuar para a célula seguinte, até todas as células estarem simuladas.

\section{Incerteza espacial}

Com as dez imagens simuladas do índice de produtividade, podemos dizer que conhecemos localmente a função de distribuição cumulativa desta variável, formada pelos dez valores simulados em cada ponto.

Para variáveis de tipo categórico (o índice é uma variável do tipo categórico com cinco modalidades), a medida de incerteza mais comum tem a designação de entropia (GoovaerTs, 1997), que pode ser definida para um conjunto de $K$ categorias do seguinte modo (em que $p_{k}{ }^{*}\left(x_{u}\right)$ é a média das dez imagens simuladas em cada célula $x_{u}$ ):

$$
H\left(x_{u}\right)=-\sum_{k=1}^{K}\left[\ln p_{k}^{*}\left(x_{u}\right)\right] p_{k}^{*}\left(x_{u}\right)
$$

O resultado desta medida é um valor entre 0 (não existe incerteza na localização das amostras) e $\ln K$ associado a uma distribuição uniforme $p_{k}{ }^{*}\left(x_{u}\right)=1 / k$. Uma medida normalizada no intervalo [0; 1] é obtida por $H_{R}\left(x_{u}\right)=H\left(x_{u}\right) / \ln K$.

Esta medida constitui um bom indicador da incerteza local e reflecte a densidade da amostragem, a variabilidade local dos valores amostrados e o modelo de variograma ajustado. 


\section{CONSTRUÇÃO DE MAPAS DO ÍNDICE DE PRODUTIVIDADE PARA O PINHEIRO-BRAVO}

Com base numa amostra cuja dimensão corresponde a 795 observações (fig. 1), para as variáveis altura dominante e idade foram calculados os valores de alguns parâmetros estatísticos básicos, ilustrados na figura 2 .

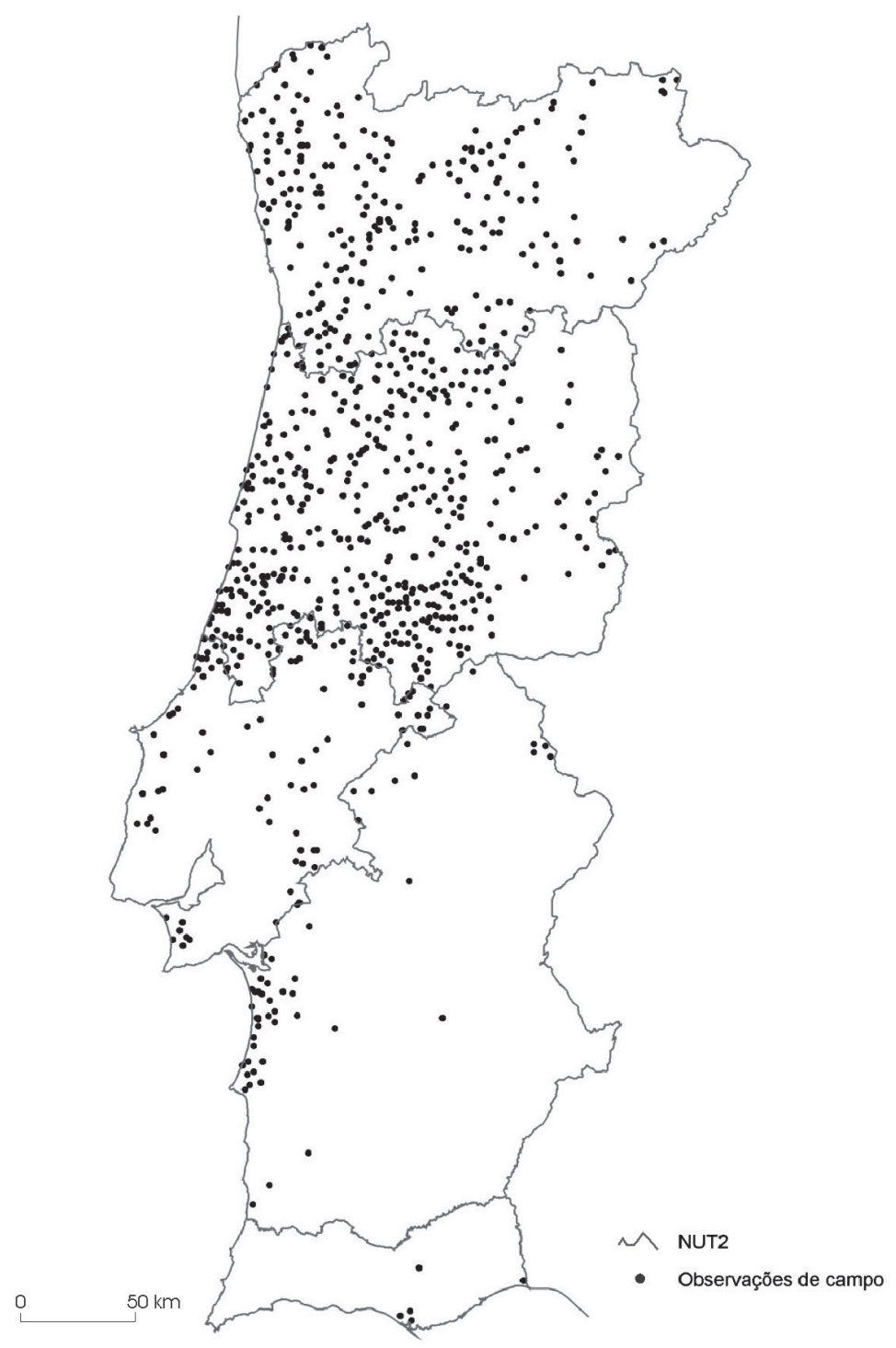

Fig. 1 - Distribuição espacial das observações de altura dominante e idade do pinheiro-bravo.

Fig. 1 - Spatial distribution of height and age measures of maritime pine. 

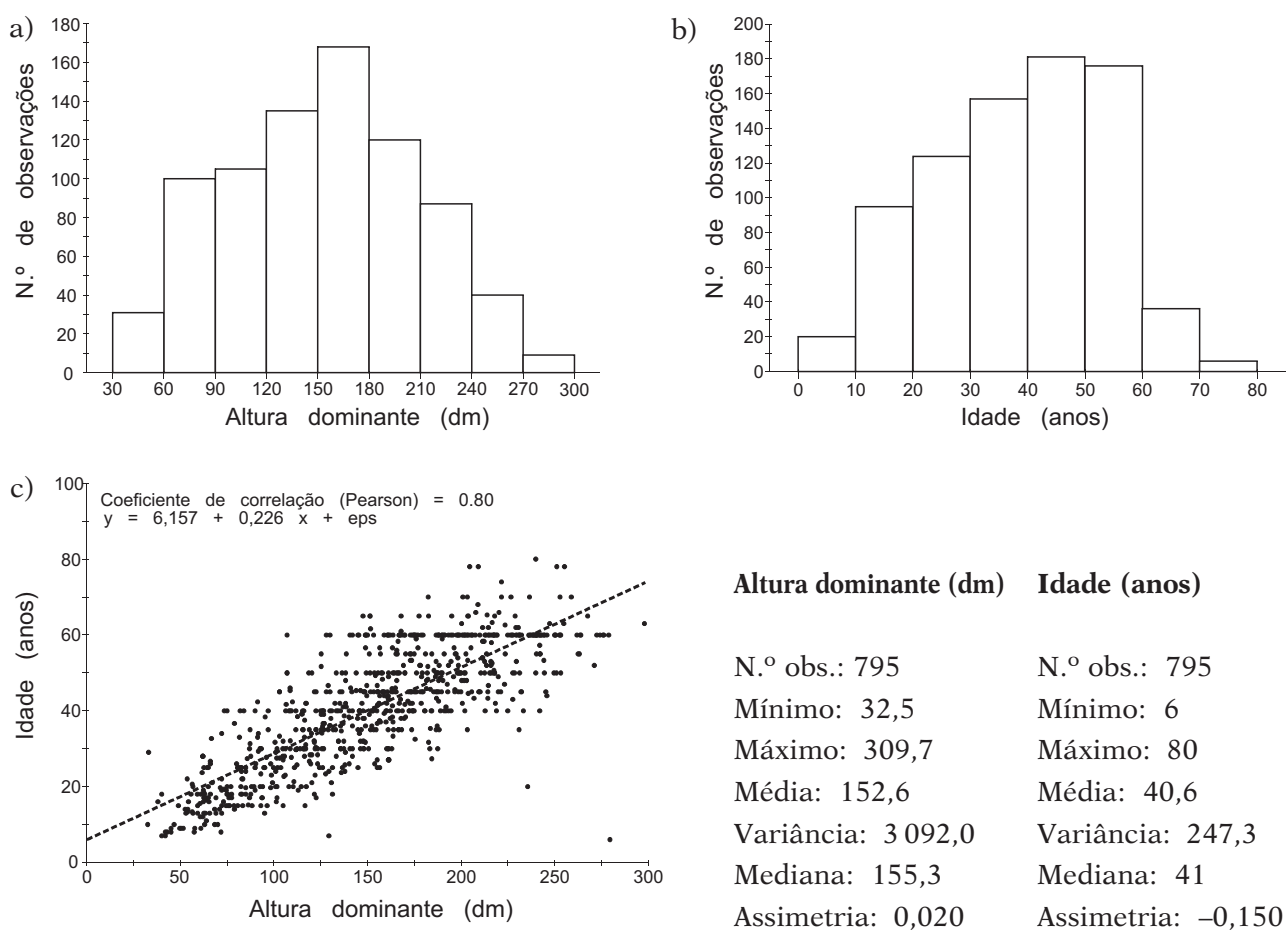

\begin{tabular}{|c|c|}
\hline Altura dominante $(\mathrm{dm})$ & Idade (anos) \\
\hline N. ${ }^{\circ}$ obs.: 795 & N. ${ }^{\circ}$ obs.: 795 \\
\hline Mínimo: 32,5 & Mínimo: 6 \\
\hline Máximo: 309,7 & Máximo: 80 \\
\hline Média: 152,6 & Média: 40,6 \\
\hline Variância: 3 092,0 & Variância: 247,3 \\
\hline Mediana: 155,3 & Mediana: 41 \\
\hline Assimetria: 0,020 & Assimetria: $-c$ \\
\hline
\end{tabular}

Fig. 2 - a) Altura dominante do pinheiro-bravo; b) idade do pinheiro-bravo e c) diagrama de dispersão entre a altura dominante e idade.

Fig. 2 - a) Maritime pine height; b) maritime pine age and c) scattergram between height and age.

Da análise da variável altura dominante verifica-se que a distribuição é aproximadamente gaussiana (assimetria próxima de 0); o maior número de árvores encontra-se na classe 150-180dm (média igual a 152,6dm), encontrando-se na amostra poucas árvores acima dos $240 \mathrm{dm}$. Quanto à variável idade, constata-se que existem poucas árvores novas (com idades até 10 anos), verificando-se as maiores ocorrências de idades entre os 40 e os 60 anos. A correlação entre as variáveis altura dominante e idade é elevada (coeficiente de correlação de Pearson igual a 0,80 ).

Para cada uma destas duas variáveis foram calculados variogramas experimentais ${ }^{7}$ e ajustados modelos teóricos de variograma de tipo esférico $(\mathrm{Sph}) \mathrm{com}$ duas estruturas (figs. 3 e 4). A variável idade apresenta um variograma isótropo, enquanto que a altura dominante apresenta um variograma anisótropo, em que a direcção principal ou de maior continuidade é E-W. Esta anisotropia é apenas

7 O variograma é uma ferramenta importante da geoestatística que permite medir a correlação de uma variável no espaço ou no tempo. 
detectada para a primeira estrutura, ou seja, para distâncias curtas (inferiores a $10000 \mathrm{~m})$.

a) (h)

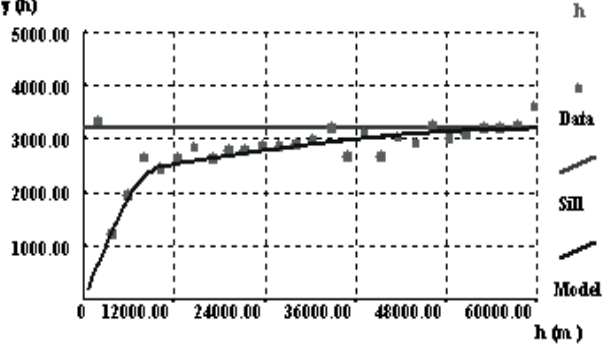

b) y (h)

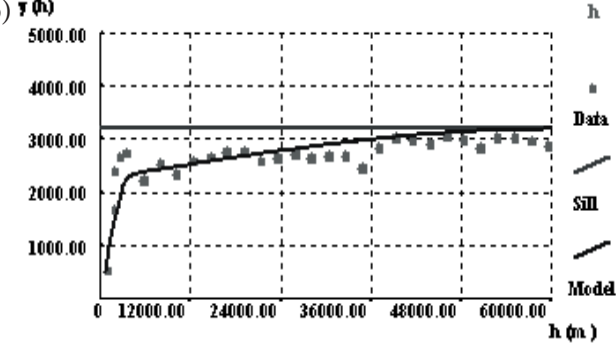

Fig. 3 - Variogramas experimentais da variável altura dominante - modelo anisótropico: a) direcção principal E-W: $\gamma(\mathrm{h})=2275 \mathrm{Sph}(10000 \mathrm{~m})+975 \mathrm{Sph}(60000 \mathrm{~m})$

e b) direcção secundária N-S: $\gamma(\mathrm{h})=2275 \mathrm{Sph}(4000 \mathrm{~m})+975 \mathrm{Sph}(60000 \mathrm{~m})$.

Fig. 3 - Experimental variograms of height-anisotropic model: a) main direction $E-W: \gamma(h)=2275 \operatorname{Sph}(10000 \mathrm{~m})+975 \operatorname{Sph}(60000 \mathrm{~m})$ and $b)$ secondary direction $N-S: \gamma(h)=2275 \operatorname{Sph}(4000 \mathrm{~m})+975 \operatorname{Sph}(60000 \mathrm{~m})$.

a) 1 (h)

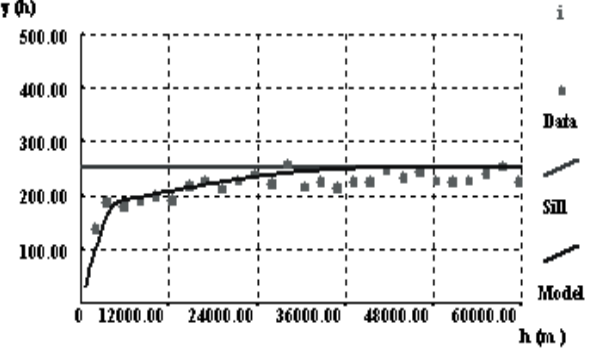

b) $($ h)

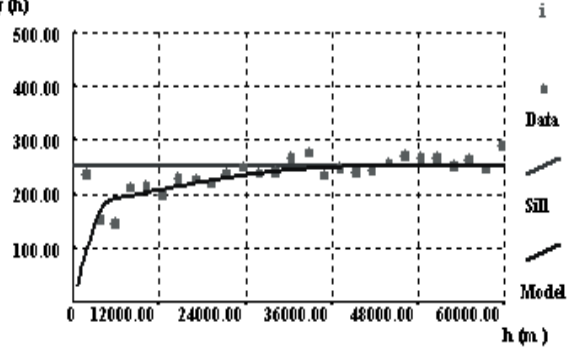

Fig. 4 - Variogramas experimentais da variável idade - modelo isótropico $\gamma(\mathrm{h})=182 \mathrm{Sph}(5000 \mathrm{~m})+78 \mathrm{Sph}(40000 \mathrm{~m})$ : a) direcção N-S e b) direcção E-W.

Fig. 4 - Experimental variograms of age - isotropic model $\gamma(h)=182 \operatorname{Sph}(5000 \mathrm{~m})+78 \operatorname{Sph}(40000 \mathrm{~m}):$ a) direction $N-S$ and b) direction $E-W$.

Foram, então, construídas dez imagens equiprováveis da altura dominante e da idade para todo o país. A figura 5 ilustra o exemplo de um par de imagens simuladas de cada variável, antes de se efectuar a sobreposição com as áreas de ocorrência de pinheiro-bravo em Portugal. Cada par de imagens simuladas de altura e idade (figs. 5a e 5b) permite calcular uma imagem simulada do índice de produtividade, utilizando as equações (1) e (2) apresentadas anteriormente (fig. 5c). A combinação de imagens e sobreposição com a mancha de pinheiro-bravo em Portugal foi feita no software ArcGIS da ESRI.

Para validação das imagens simuladas são, igualmente, representados os valores de alguns parâmetros estatísticos básicos dessas imagens e o diagrama de dispersão entre a altura dominante e a idade (fig. 6). 

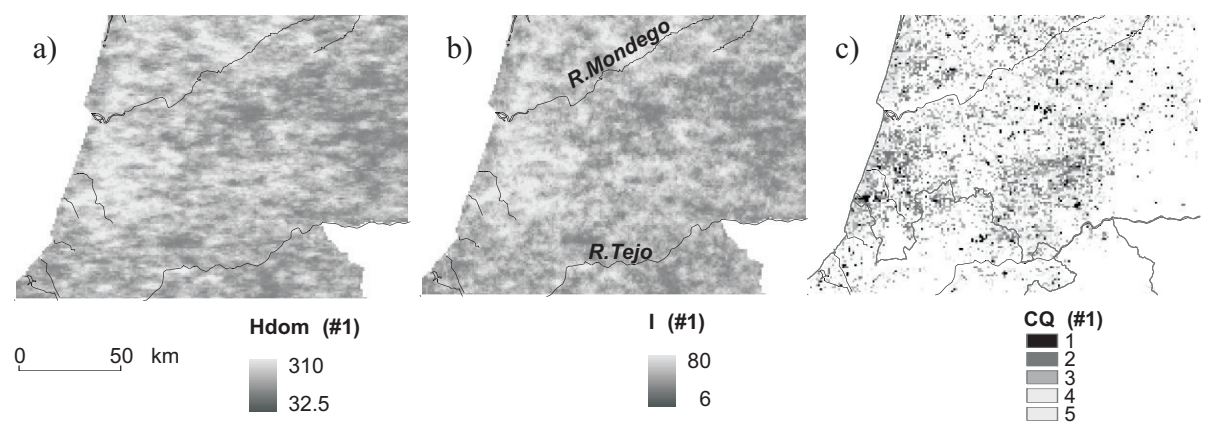

Fig. 5 - Exemplo dos resultados de uma simulação: a) Imagem simulada da altura dominante; b) Imagem simulada homóloga da idade e c) Imagem calculada do índice de produtividade.

Fig. 5 - Simulation results example: a) simulated image of height; b) corresponding simulated image of age and c) derived image of the productivity index.
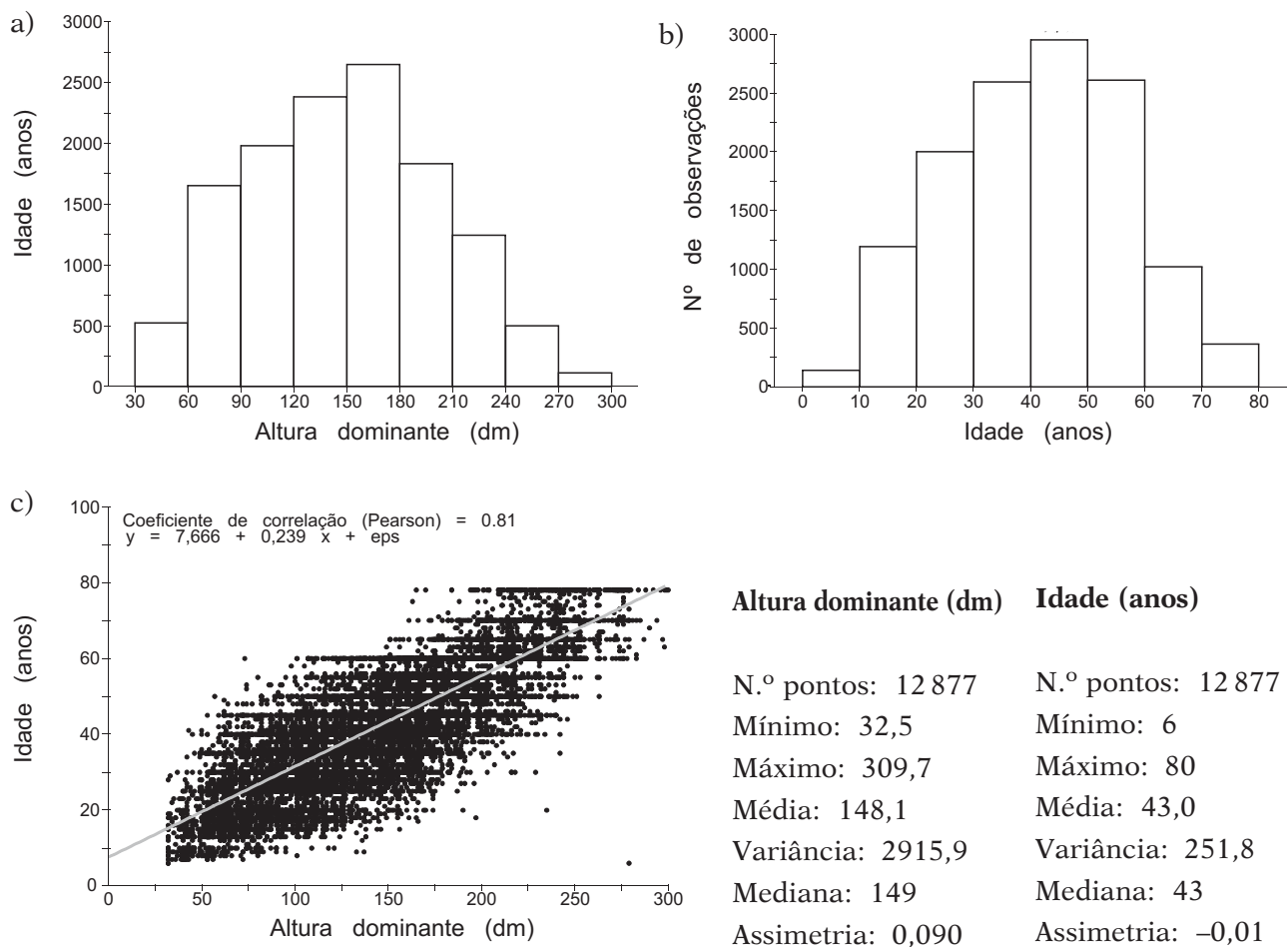

\begin{tabular}{|c|c|}
\hline Altura dominante $(\mathrm{dm})$ & Idade (anos) \\
\hline N. ${ }^{\circ}$ pontos: 12877 & N. ${ }^{\circ}$ pontos: 12877 \\
\hline Mínimo: 32,5 & Mínimo: 6 \\
\hline Máximo: 309,7 & Máximo: 80 \\
\hline Média: 148,1 & Média: 43,0 \\
\hline Variância: 2915,9 & Variância: 251,8 \\
\hline Mediana: 149 & Mediana: 43 \\
\hline & Assimetria: -0 , \\
\hline
\end{tabular}

Fig. 6 - Exemplo dos resultados de uma simulação: a) altura dominante do pinheiro-bravo; b) idade do pinheiro-bravo e c) diagrama de dispersão entre a altura dominante e idade.

Fig. 6 - Simulation results example: a) maritime pine height; b) maritime pine age and c) scattergram between height and age. 
A partir de dez imagens simuladas de índice de produtividade (variável de tipo categórico) foi calculada uma imagem de probabilidades, em que, a cada célula, é alocada a probabilidade de pertença a cada uma das categorias $X_{k}(k=1, K)$. Finalmente, este mapa de probabilidades foi transformado num mapa final classificado, que corresponde à imagem mais provável do índice de produtividade. A classificação foi baseada nas probabilidades locais e globais de cada uma das células pertencer a cada uma das categorias $X_{k}(k=1, K)$ (SOARES, 1992; Almeida et al., 1993). Na figura 7, encontra-se representado o mapa final do índice de produtividade do pinheiro-bravo em Portugal (as áreas de melhor produtividade aparecem a verde escuro e as de menor a amarelo) e, na figura 8 o respectivo mapa de incerteza. Tendo em conta a metodologia, este mapa, resulta da incerteza combinada das varáveis altura dominante e idade.
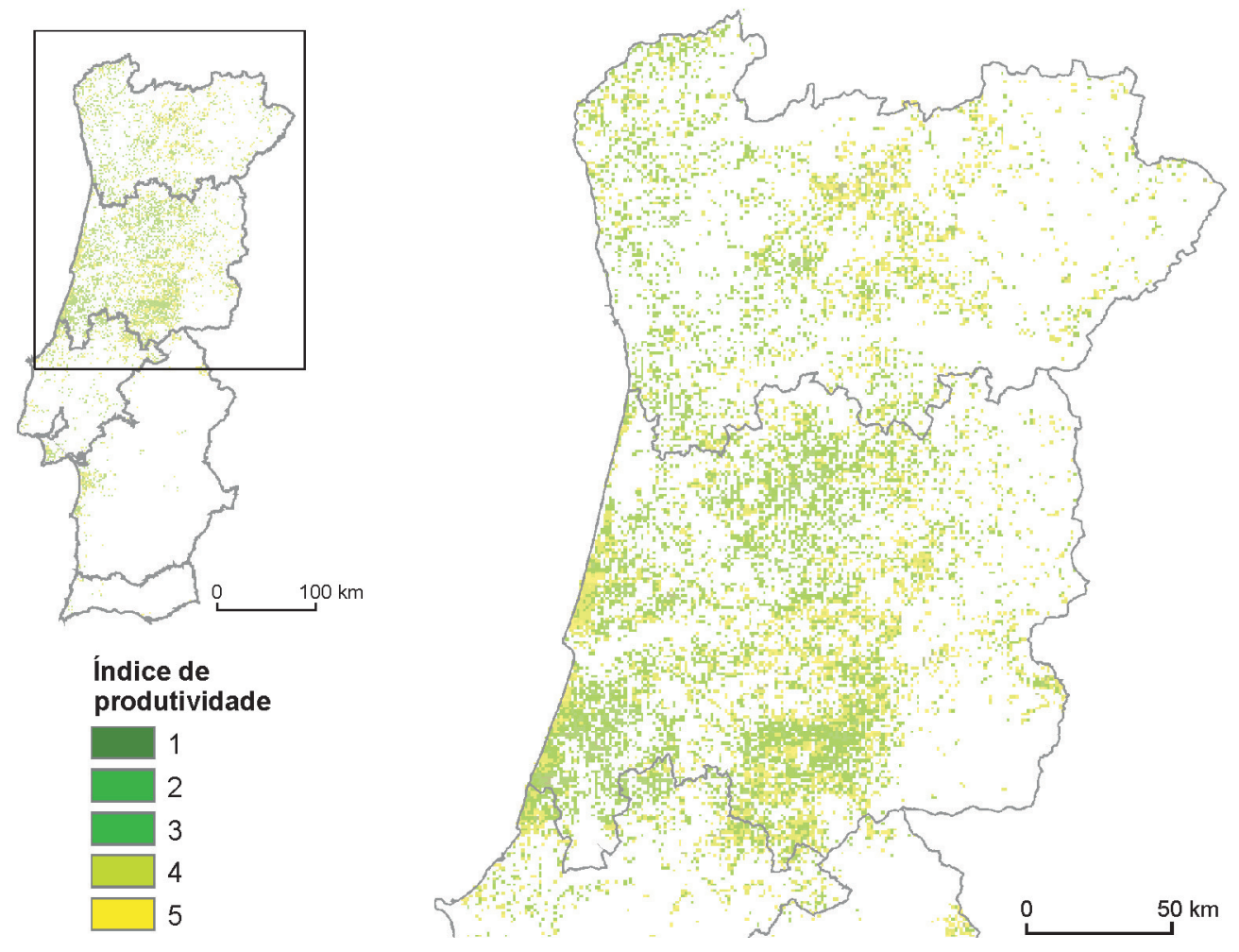

Fig. 7 - Índice de produtividade do pinheiro-bravo e pormenor nas NUT II Norte e Centro.

Fig. 7 - Productivity index of the maritime pine and zoom on the NUT II North and Centre.

A figura 7 mostra claramente que as melhores áreas de produtividade para o pinheiro-bravo (verde escuro) estão relativamente afastadas do Litoral e 
correspondem a regióes onde, provavelmente, ocorrem os solos mais produtivos em regiões climáticas favoráveis. Perto do Litoral, a floresta de pinheiro-bravo é a ocupação predominante, apresentando, no entanto, uma fraca produtividade (terrenos dunares). Nas zonas de montanha, o índice de produtividade para o

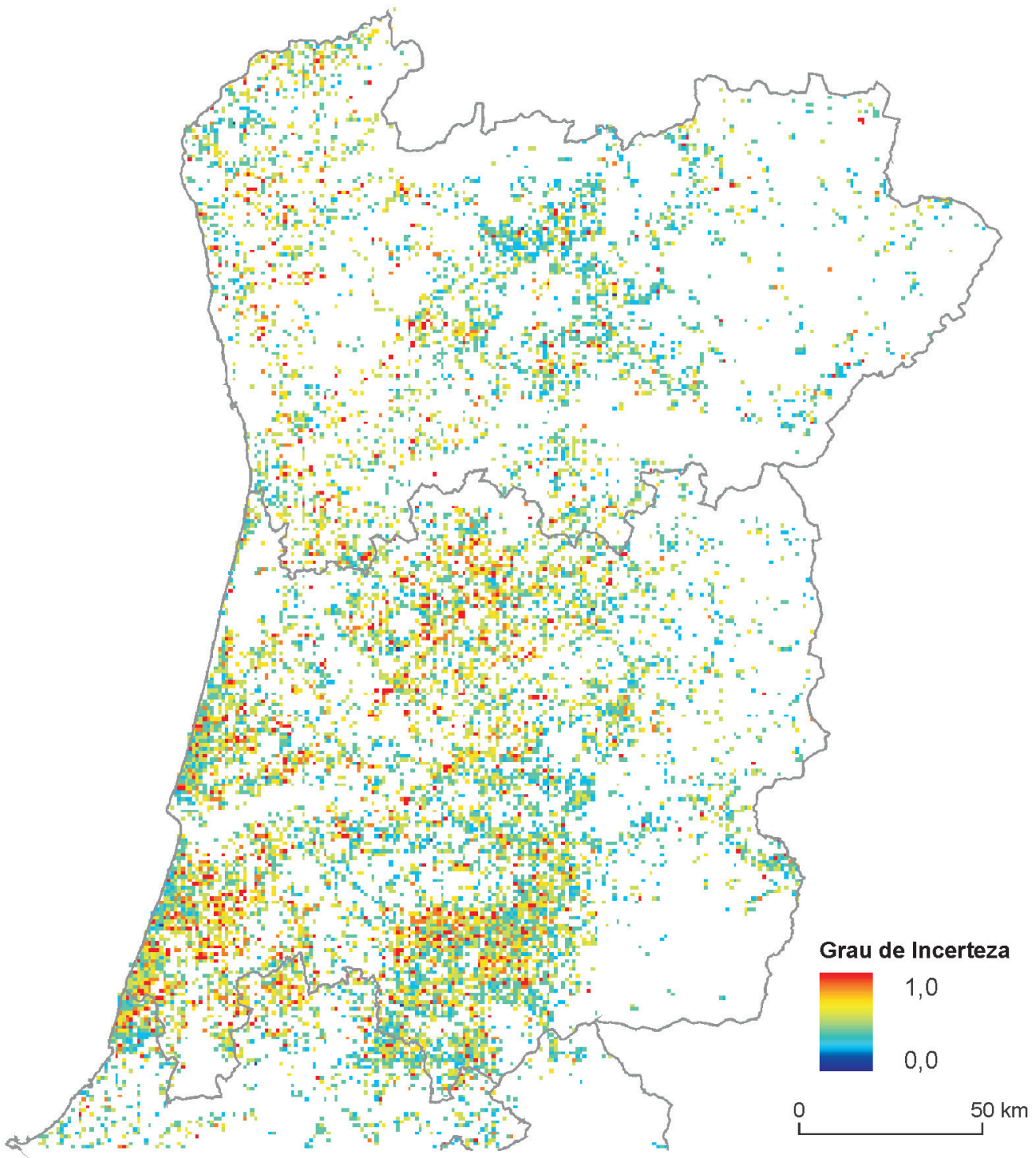

Fig. 8 - Distribuição da incerteza local na avaliação do índice de produtividade (pormenor nas regiões Norte e Centro).

Fig. 8 - Local uncertainty with regard to the productivity index (zoom on the North and Centre areas). 
pinheiro-bravo tem um gradiente variável, aparecendo as melhores áreas na zona da Serra da Gardunha (Oleiros, Mação e Sertã). Na NUT II do Pinhal Interior Norte (que inclui concelhos como Lousã, Tábua, Góis e Pampilhosa da Serra), o índice de produtividade é relativamente bom. Estas zonas apresentam uma combinação de factores propícios ao desenvolvimento do pinheiro-bravo, sendo os mais determinantes os solos e o clima. A altitude e a ocorrência de alguns factores bióticos e abióticos, como os fogos e as pragas, revelam-se igualmente importantes na obtenção destes valores.

Analisando a figura 8 , verifica-se uma grande heterogeneidade na dispersão do grau de incerteza, isto é, a ocorrência de valores de máxima incerteza próximos de valores de baixa incerteza. A razão principal decorre da existência de povoamentos de pinheiro bravo de estruturas irregulares (por exemplo, co-ocorrência de várias classes de idade no mesmo local, ciclos de fogos, pragas, etc.) que depois são reflectidas no modelo ajustado dos variogramas experimentais de ambas as variáveis que apresentam sempre uma primeira estrutura com amplitude baixa (inferior a $10000 \mathrm{~m}$ ). Esta distância corresponde à influência máxima de cada medição, verificando-se que propagação da influência de cada amostra é reduzida.

\section{CONCLUSÕES}

A metodologia apresentada neste trabalho permitiu produzir mapas, a nível nacional, de um índice de produtividade para o pinheiro-bravo distribuído por cinco classes, baseada na informação recolhida nos levantamentos de campo da 3. ${ }^{\mathrm{a}}$ Revisão do Inventário Florestal Nacional.

Este estudo apresentou como principal componente inovadora o recurso a modelos de Simulação e Co-Simulação geoestatística, aplicados aos recursos florestais. Estes métodos de simulação poderão dar grande contributo na aferição da malha de amostragem necessária à recolha de informação em futuros trabalhos de campo, uma vez que a incerteza dos modelos de caracterização espacial não dependem exclusivamente da distribuição espacial das amostras, mas também da variabilidade local. A simulação geoestatística possibilita a caracterização da incerteza local face à malha de amostragem e à variabilidade das medições (por exemplo, a variância de krigagem apenas transporta a incerteza proveniente da geometria da malha de amostragem), sendo por isso uma ferramenta importante na programação de futuras campanhas de amostragem, face ao detalhe requerido num inventário florestal.

\section{BIBLIOGRAFIA}

Almeida, J.; Soares, A. e Reynaud R. (1993) - Modelling the shape of several marble types in a quarry. Proceedings of the XXIV International Symposium APCOM (Vol. 3). Montreal: 452-459.

Alves, A. (1988) - Técnicas de produção florestal, 2. edição. INIC, Lisboa. 
Direcção-Geral das Florestas (2001) - Inventário florestal nacional de Portugal Continental. 3. ${ }^{a}$ Revisão 1995-1998, Relatório Final. DGF, Lisboa. 233 pp.

Goovaerts, P. (1997) - Geostatistics for natural resources evaluation. Oxford University Press, New York.

Oliveira, A.; Pereira J. e Correia A. (2000) - A silvicultura do pinheiro bravo. Centro Pinus, Porto.

SoAREs, A. (1992) - Geostatistical estimation of multi-phase structures. Mathematical Geology, 24(2): 149-160.

SoARes, A. (2000) - Geoestatística para as ciências da terra e do ambiente. Colecção Ensino da Ciência e Tecnologia, n. ${ }^{\circ}$ 9. IST Press, Lisboa.

SoAres, A. (2001) - Direct sequential simulation and co-simulation. Mathematical Geology, 33(8): 911-926. 